\title{
Heterogeneity, Inherent and Acquired Drug \\ Resistance in Patient-Derived Organoids Models of Primary Liver Cancer
}

Guang-Wen Cao ( $\sim$ gcao@smmu.edu.cn )

Linfeng Xian

Pei Zhao

Xi Chen

Zhimin Wei

Hongxiang Ji

Jun Zhao

Donghong Liu

Zishuai Li

Wenbin Liu

Xue Han

Youwen Qian

Hui Dong

Xiong Zhou

Junyan Fan

Xiaoqiong Zhu

Jianhua Yin

Xiaojie Tan

Dongming Jiang

Hongping Yu

Article

Keywords: primary liver cancer, organoids, sorafenib resistance, cancer stem cell, epithelialmesenchymal transition

Posted Date: January 10th, 2022

DOI: https://doi.org/10.21203/rs.3.rs-1229184/v1

License: () (1) This work is licensed under a Creative Commons Attribution 4.0 International License. Read Full License 


\section{Abstract}

To screen for sensitive drugs for recurrent primary liver cancer (PLC) and elucidate the mechanisms underlying inherent and acquired drug resistance, we established a platform of organoids, organoidsderived xenograft (ODX) mouse models, and patient-derived xenograft (PDX) mouse models of primary liver cancer (PLC). Fifty-two organoids were established from 153 PLC patients. Establishing organoids of hepatocellular carcinoma (HCC) displayed a trend toward a higher success rate than establishing PDX $(29.0 \%$ vs. $23.7 \%)$ and took a shorter duration ( $13.0 \pm 4.7$ vs. $25.1 \pm 5.4$ days, $P=2.28 \times 10-13)$ than establishing PDX models. Larger tumor, vascular invasion, and advanced stage were significantly associated with successful establishment of organoids and PDX in HCC. Organoids and ODX recapitulated PLC histopathological features but enriched more aggressive cell types. PLC organoids were mostly resistant to lenvatinib in vitro but sensitive in ODX model, indicating innate immunity plays a role. Acquired sorafenib-resistant HCC organoids were generated via 3-5 months of induction. RNAsequencing indicated that stemness- and epithelial-mesenchymal transition (EMT)-related gene sets were upregulated, whereas liver development- and liver specific molecule-related gene sets were downregulated, in acquired sorafenib-resistant organoids. Targeting mTOR signaling pathway was effective in treating acquired sorafenib-resistant HCC, possibly via inducing phosphorylated S6 kinase. Genes upregulated in acquired sorafenib-resistant HCC organoids were often associated with unfavorable prognosis. Conclusively, HCC organoids perform better than PDX for drug selection. Acquired sorafenib resistance in organoids promotes HCC aggressiveness via facilitating the stemness, retrodifferentiation, and EMT. Phosphorylated S6 kinase might be predictive for drug resistance in HCC.

\section{Introduction}

Primary liver cancer (PLC) is the sixth most commonly diagnosed cancer and the third leading cause of cancer death worldwide ${ }^{1}$. In China, PLC remains the second cause of cancer death. The age-standardized mortality rate is higher in the 40-64-year-old population than in the $\geq 65$-year-old population ${ }^{2}$. The major histotypes of PLC are hepatocellular carcinoma (HCC) (comprising 94.6\%) and intrahepatic cholangiocarcinoma (ICC) (3.7\%), and combined hepatocellular cholangiocarcinoma (CHC) $(1.7 \%)$ in Eastern $\mathrm{China}^{3}$. Surgical resection is the mainstay of curative treatment. Frequent postoperative recurrence is the major obstacle to improve the prognosis. Approximately $70 \%$ of HCC patients relapse within five years after hepatectomy, the incidence of post-liver transplantation recurrence remains 10$20 \%$, 5 . Effective treatments for postoperative recurrence are extremely lacking.

Effective therapeutic options for PLC recurrence are hindered by the shortage of reproducible and reliable human models. Tumor-derived cell lines have long been applied to study the underlying biologic processes and as platforms for discovering and evaluating anticancer therapeutics ${ }^{6}$. Although tumor cell lines have allowed pioneering advances in cancer biology, they might not recapitulate critical characteristics and the heterogeneity of PLC in situ. Recently, PLC patient-derived tumor organoids have been developed ${ }^{7-11}$. These organoids have been proven to retain the morphology, genetic heterogeneity, 
and expression pattern of the original tumor markers of PLC. These organoids not only help elucidate the molecular mechanism of oncogenesis but also provide an opportunity of drug screening for precision treatment.

However, several key issues remain to be addressed to construct a platform of PLC organoids for precision medicine. First, the factors affecting the success rate of establishing organoids are not established, due to small sample size of surgically removed tissues applied in each study. The numbers of patients from whom the organoids developed were eight ${ }^{7}$, eleven ${ }^{8}$, five $^{9}$, four $^{10}$, and seven ${ }^{11}$ in

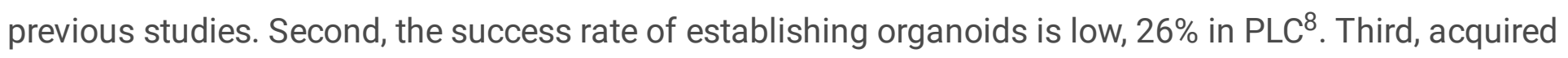
drug resistance reflecting the evolution of PLC has not been evaluated in the organoids, in which the heterogeneity should be distinct from that in cell lines ${ }^{12}$. Here, we successfully established 56 tumor organoids from 160 patients and evaluated the factors affecting developing the organoids in HCC. We demonstrated that PLC organoids recapitulated the histological features of the original tumor but enriched aggressive cell types in vitro and in vivo. We also generated acquired sorafenib-resistant HCC organoids and identified that targeting mTOR signaling was effective in treating HCC with sorafenib resistance. This study not only provides intact technology to establish organoid platform for drug selection but also elucidates the mechanism by which PLC evolves under sorafenib pressure.

\section{Results}

\section{Establishment of tumor organoids and patient-derived xenograft (PDX) models}

Tumor organoids were cultured using surgically removed primary tumor tissues of 160 patients (131 with HCC, 17 with ICC, five with CHC, one with hepatoblastoma, one with hepatocellular adenoma, one with B cell lymphoma, one with hepatocellular carcinoma with bile duct epithelial differentiation, one with hepatic sarcoma; one with angiomyolipoma and one with metastatic carcinoma) from January 2018 to January 2019, from which we successfully constructed organoids from the tissues of 38 patients with HCC (29.0\%), nine with ICC (52.9\%), five with CHC, one with hepatoblastoma, one with hepatocellular adenoma, one with angiomyolipoma, and one with metastatic carcinoma (Figure S1A). The organoids from HCC and ICC were frozen in liquid nitrogen and then thawed for in vitro culture for several (3-5) cycles. This process lasted for up to 3.5 years, which did not alter their histological features. We also successfully developed PDX models using these tissues from 31 patients with $\mathrm{HCC}(23.7 \%)$, seven with ICC (41.2\%), three with $\mathrm{CHC}$, one with hepatoblastoma, one with hepatocellular adenoma, and one with metastatic carcinoma (Figure S1B). The duration of HCC organoids' culture from tissue separation to the first passage was significantly shorter than that of PDX development from the first tumor implantation to the second implantation ( $13.0 \pm 4.7$ vs. $25.1 \pm 5.4$ days, $P=2.28 \times 10^{-13}$ ) (Figure S1C,1D).

\section{The factors affecting the success rate of developing organoids and PDX models}

To identify factors influencing the success rate of constructing HCC organoids, we compared the clinical data of patients whose tissues were successfully developed to organoids with those whose tissues 
failed. It was found that larger tumor size $(P=0.030)$, microvascular invasion (MVI) $(P=0.014)$, macrovascular invasion $(P<0.001)$, advanced TNM stage $(P=0.006)$, and advanced Barcelona Clinic Liver Cancer (BCLC) stage $(P=0.010)$ were associated with successful development of HCC organoids (Table 1). Larger tumor size $(P=0.005), \mathrm{MVI}(P=0.014)$, macrovascular invasion $(P=0.001)$, advanced TNM stage $(P=0.004)$, and advanced BCLC stage $(P=0.010)$ were also associated with successful PDX establishment (Table S1).

\section{PLC organoids and PDX recapitulate the histopathological features of the original PLC types in vitro and in animal models}

We confirmed that tumor organoids recapitulate histological features of the original tumors. HCC organoids mostly displayed solid structures. ICC organoids formed compact spheroids and irregularly shaped cyst-like structures. To assess if tumor organoids retained the histological characteristics of the corresponding PLC subtypes in vivo, we subcutaneously transplanted the organoids of HCC, ICC, and CHC into nude mice to establish organoids-derived xenograft (ODX). It was shown that ODX retained the histological characteristics of the corresponding PLC subtypes in nude mice. Moreover, PDX in nude mice also well recapitulated the histology of the corresponding PLC subtypes. These data are shown in Figure 1.

\section{Tumor organoids enriched the aggressive cell types of the original PLC subtypes, but retain the heterogeneity}

Immunohistochemistry demonstrated that a-fetoprotein (AFP) was highly expressed in HCC organoids, rather than in ICC and $\mathrm{CHC}$ organoids, which was consistent with the expression pattern of the original tumors (Figure S2A). The expression of cytokeratin 19 (CK19), a marker of HCC stem cells, was higher in tumor organoids than in the corresponding tumor tissues of HCC-25 (immunoreactive score: 12.0 vs. 1.2, $\left.P=8.1 \times 10^{-9}\right)$, ICC-6 (12 vs. $\left.7.5, P=0.015\right)$, and $\mathrm{CHC}-3\left(10.2\right.$ vs. $\left.2.8, P=3.0 \times 10^{-5}\right)$, rather than in that from HCC-118. The expression of CK19 was higher in ODX than in the corresponding tumor tissues of HCC-25 (9.2 vs. $1.2, P=4.0 \times 10^{-4}$ ) and $\mathrm{CHC}-3$ (8.2 vs. $2.8, P=0.001$ ), rather than in those from HCC-118 and ICC-6. The expression of CK19 was not statistically different in the primary tumors and PDX of HCC-25, HCC118, ICC-6, and CHC-3 (Figure S2B). The expression of epithelial cell adhesion molecule (EpCAM), another epithelial stem marker, was higher in tumor organoids of HCC-25 (8.8 vs. $\left.2.8, P=2.8 \times 10^{-6}\right)$ and $\mathrm{CHC}-3(3.7$ vs. $1.7, P=0.023)$ as well as the ODX from HCC-25 ( 8.3 vs. $\left.2.8, P=9.8 \times 10^{-6}\right)$ than in the corresponding tumor tissues. No difference was found between the organoids and primary tumors of HCC-118 and ICC-6 as well as between PDX and the corresponding tumor tissues from HCC-25, HCC-118, ICC-6, and CHC-3 (Figure S2C). These results indicate that tumor organoids enriched the aggressive cell types of the corresponding PLC subtypes; however, the heterogeneity is evident.

\section{Heterogeneity of inherent drug response in tumor organoids}

To evaluate if tumor organoids are suitable for drug selection in vitro, we treated PLC organoids with different concentrations of sorafenib, regorafenib, lenvatinib, and mTOR inhibitor compound 
RAD001/TAK228 / phenformin (RTP) and then examined cell viability. It was found that sorafenib and regorafenib inhibited the growth of HCC organoids; however, this effect was not observed in nearly all the ICC and CHC organoids. RTP decreased the growth of PLC organoids in a dose-dependent manner. However, the majority of the PLC organoids showed resistance to lenvatinib treatment, with an halfmaximal inhibitory concentration $\left(\mathrm{IC}_{50}\right)$ value higher than the maximum screening concentration (Figure $2 A, B)$.

To explore the therapeutic potentials of targeted therapeutics including RTP on PLC of each histotype, we examined their effects on the growth of tumor ODX models. The results show that the anti-tumor effect of RTP was comparable to that of sorafenib in HCC, while RTP inhibited ICC and CHC more effectively than did sorafenib. In contrast to the resistance to the majority of PLC organoids, lenvatinib was effective in all the tested ODX models (Figure 2C,D).

\section{Acquired sorafenib-resistant HCC organoids and gene expression profiles}

To explore the evolution of the resistance to sorafenib in HCC organoids, four HCC organoids (HCC-10, HCC-25, HCC-52, and HCC-118) were randomly selected. HCC-10 was derived from a female HBV-positive HCC patient with lymph node metastasis. HCC-25 was an invasive tumor organoid derived from a female HBV-positive HCC (HBV-HCC) patient. HCC-52 was an invasive tumor organoid derived from a male HBsAg-negative HCC patient. HCC-118 was a non-invasive tumor organoid derived from a male HBVpositive HCC patient who had received long-term antiviral treatment before surgery. After approximately 3-5 months of culture, the $\mathrm{IC}_{50}$ values of drug-resistant HCC-10, HCC-25, HCC-52, and HCC-118 strains increased 1.59-, 4.11-, 2.01-, and 2.31-fold, respectively (Table S2).

RNA sequencing was applied to assess the expression profiles of mRNA between sorafenib-resistant and parental tumor organoids from four HCC patients (HCC-10, HCC-25, HCC-52, and HCC-118). Combining the data from the four organoids, we identified that a group of genes including MCM6 and RRS1 were upregulated while most genes including TP53INP2 and MYH14 were downregulated in acquired sorafenib-resistant HCC organoids (Table S3). GSEA identified a group of gene sets enriched in acquired sorafenib-resistant organoids (Table S4). Importantly, cancer stemness-related gene sets including Mycand EGFR-related gene sets were enriched in the sorafenib-resistant organoids; epithelial-mesenchymal transition (EMT)-related gene sets including TGF 31 - and E2F-related gene sets were also enriched in the sorafenib-resistant organoids (Figure 3A,B). Importantly, liver development- and liver specific gene-related gene sets were often downregulated, whereas undifferentiated cancer- and proliferation-related gene sets were upregulated in the sorafenib-resistant organoids (Figure 3C,D).

Although the sorafenib-resistant organoids enriched with gene signatures of stemness and EMT, the heterogeneity among the four HCC organoids was still evident. Sorafenib-resistant HCC-25, HCC-52, and HCC-10 were associated with upregulation of stemness- and EMT-related gene sets, whereas sorafenibresistant HCC-118 was associated with downregulation of stemness- and EMT-related gene sets (Figure S3). We then evaluated the effect of drug resistance on the expression of stem-related genes in our 
sorafenib-resistant HCC organoids by either Western blot or immunohistochemistry. Epithelial markers Ecadherin and ZO-1 were downregulated in sorafenib-resistant HCC-25, HCC-10, and HCC-52 compared to their parental counterparts, in contrast to $\mathrm{HCC}-118$, while $\beta$-catenin was upregulated in sorafenib-resistant HCC-25 and HCC-10 (Figure 4A). Stem cell markers CD133, CK19, and $\beta$-catenin were upregulated in sorafenib-resistant HCC-25 compared to its parental HCC-25 and this was not apparent in HCC-118 (Figure 4B). The expression of ABCG2 was upregulated in sorafenib-resistant HCC-118 and HCC-52 compared to their parental counterparts; the expression of EpCAM was upregulated in sorafenib-resistant HCC-118 and HCC-10 compared to their parental counterparts. Claudin-1 and N-cadherin were upregulated in sorafenib-resistant HCC-10 compared to its parental counterpart, while CD44 was upregulated in sorafenib-resistant HCC-25 compared to its parental counterpart by both Western blot and IHC assays (Figure S4). Immunohistochemistry for other organoids was technically unsuccessful. Thus, induction of sorafenib resistance is related to the process of cancer stemness and EMT/partial EMT. Similarly, the heterogeneity is apparent.

\section{Targeting mTOR signaling pathway was effective in treating acquired sorafenib-resistant HCC organoids}

After establishing acquired sorafenib-resistant HCC organoids, we sought to investigate if inhibitors targeting the sorafenib-induced signaling pathways could inhibit the growth of sorafenib-resistant HCC organoids. We tested the effects of available inhibitors on the cell viability of sorafenib-resistant HCC organoids. It was found that RTP was effective in inhibiting sorafenib-resistant HCC-25 and HCC-118 organoids, with $\mathrm{IC}_{50}$ values of 1.918 and $1.828 \mathrm{nM}$, respectively (Figure 5A,B). However, the expression levels of mTOR signaling molecules were not significantly upregulated in sorafenib-resistant HCC-25 and HCC-118 organoids (Table S3). It was found that phosphorylation of S6, a key downstream target of $\mathrm{PI} 3 \mathrm{~K} / \mathrm{AKT} / \mathrm{mTOR}$ pathway ${ }^{13}$, was apparently upregulated in sorafenib-resistant HCC-25 and HCC-118 organoids (Figure 5C). Thus, phospho-S6 should be predictive in sorafenib-resistant HCC.

As acquired sorafenib-resistant HCC-25 organoids exhibited more stemness than did acquired sorafenibresistant HCC-118 (Figuire 4B) and the HCC-118 organoids responded to RTP more efficiently than did the HCC-25 (Figure 5A,B), we sought to identify factor related to the difference. Interestingly, we found that the expression of $\beta$-actin was apparently downregulated in acquired sorafenib-resistant HCC-25 (Figure 5D). We then removed sorafenib and cultured sorafenib-resistant HCC-25 organoids for an additional two weeks, and the expression of $\beta$-actin was restored after sorafenib withdrawal (Figure 5E). However, the expression of $\beta$-actin did not differ between the parental and sorafenib-resistant HCC-118 (Figure 5F). These data imply that $\beta$-actin protein is not only indispensable for efficient cell migration ${ }^{14}$, but also has an opposite effect on maintaining cancer stemness.

\section{Molecules upregulated in sorafenib-resistant organoids are often associated with unfavorable prognosis in $\mathrm{HCC}$}

The association of the differential expressed genes related to sorafenib-resistance with postoperative prognosis was evaluated using the data from the Cancer Genome Atlas (TCGA) database. The genes 
whose expressions were dysregulated in the sorafenib-resistant HCC organoids are listed in Table S5. Of the 26 OS-related genes upregulated in the sorafenib-resistant HCC organoids, 21 were significantly associated with unfavorable prognosis. The genes upregulated in the sorafenib-resistant HCC organoids were more related to unfavorable OS than were the downregulated genes $(21 / 26$ (86.8\%) vs. $61 / 119$ (51.3\%), $P=0.010)$; the genes upregulated in the sorafenib-resistant HCC organoids were more related to unfavorable DFS than were the downregulated genes (12/16 (75.0\%) vs. 36/95 (37.9\%), $P=0.012$ ) (Figure S5). These genes might reflect $\mathrm{HCC}$ evolution and development during generating drug resistance against sorafenib pressure.

\section{Discussion}

In this study, we established tumor organoids and PDX of each PLC histotype from 160 patients. The mean duration of establishing tumor organoids was significantly shorter than that of establishing PDX model; furthermore, establishing tumor organoids displayed a trend toward a higher success rate than establishing PDX (HCC: $29.0 \%$ vs. $23.7 \%$; ICC: $52.9 \%$ vs. $41.2 \%$ ). These data indicate that tumor organoids perform better than PDX as the platform to select drug for the treatment of PLC recurrence. Although tumor organoids and PDX recapitulate the histopathological features of the original PLC types in vitro and in vivo, more cancer stem cell markers were identified in tumor organoids than in PDX models. For the first time, we demonstrated that larger tumor size, the presence of vascular invasion, advanced TNM stage, and advanced BCLC stage were associated with successful development of tumor organoids and PDX models. These factors are significantly associated with unfavorable postoperative prognosis ${ }^{3-5,15}$, indicating that aggressive tumor cell subpopulations of PLC may have growth advantage in tumor organoid. Cancer stemness and EMT are often associated with the aggressiveness of $\mathrm{HCC}^{16}$. Our tumor organoids may enrich aggressive subpopulations of PLC, which is quite suitable for screening sensitive drugs.

Our data indicate that sorafenib and regorafenib can inhibit the growth of HCC organoids, which are consistent with clinical findings ${ }^{17}$. Our ICC and $\mathrm{CHC}$ organoids are resistant to regorafenib, which is not consistent with a previous PDX model ${ }^{18}$. We also demonstrated that RTP decreased the growth of all PLC organoids both in vitro and in ODX models. Thus, targeting to mTOR signaling pathway is important in treating PLC of each histotype, especially in treating ICC and CHC. Interestingly, PLC organoids were resistant to lenvatinib in vitro, but it was effective in all the tested ODX models. The antitumor efficacy of lenvatinib is mainly attributed to its antiangiogenic activity. A previous study has demonstrated that lenvatinib inhibits melanoma and renal cancer by enhancing the tumor infiltration and activation of natural killer (NK) cells ${ }^{19}$. Athymic nude mice have normal or even compensated NK activity ${ }^{20}$. Our findings indicate that tumor organoids and the ODX models might be complementary in testing drug sensitivity for PLC.

Sorafenib is the first-line targeted agent that prolongs the median survival time of patients with advanced $\mathrm{HCC}$ by nearly three months; unfortunately, most patients develop resistance to this drug within six 
month ${ }^{21}$. The mechanisms by which HCC develops resistance to sorafenib are largely unknown, although some molecular events such as Hedgehog signaling, Jak-STAT pathway, oncogene KIF14, and IGF/FGF signaling have been suggested to be related to sorafenib resistance in $\mathrm{HCC} 10,11,22,23$. Here, we induced acquired sorafenib-resistant organoids from four HCC patients following 3-5 months of culture. RNA-sequencing revealed that EMT-promoting molecules including MCM6 and $R R S 1^{24,25}$ were upregulated, while EMT-suppressing molecules including TP53INP2 and $M Y H 14^{26,27}$ were downregulated in sorafenib-resistant HCC organoids. Our GSEA showed that cancer stemness-related gene sets including Myc- and EGFR-related gene sets ${ }^{28,29}$ as well as EMT-related gene sets including TGF $\beta 1$ - and E2F-related gene sets ${ }^{30,31}$ were significantly enriched in acquired sorafenib-resistant HCC organoids. Thus, cancer stemness and EMT may contribute greatly to the development of sorafenib resistance in HCC.

The heterogeneity in expression patterns of the EMT-related and stemness-related genes were evident among HCC-118 and other three organoids. The expression of E-cadherin and ZO-1, well-established suppressors of $\mathrm{EMT}^{32}$, was downregulated in sorafenib-resistant HCC organoids except HCC-118. CD133, CK19, $\beta$-catenin, ABCG2, claudin-1, N-cadherin, and CD44, cancer stem / EMT markers ${ }^{11,32-35}$, were upregulated only in some rather than all of the sorafenib-resistant HCC organoids. This heterogeneity is solid, as the results of Western blots were quite consistent with those of IHC. This evidence supports that partial / hybrid EMT programs, defined by incomplete activation of EMT transcription factors ${ }^{31,36,37}$, play an important role in generating sorafenib resistance in HCC organoids.

After a series of tests to identify available inhibitors that can be applied to treat sorafenib-resistant HCC, we found that MTOR inhibitor compound RTP was effective for the sorafenib-resistant HCC organoids. Further study revealed that phosphorylated S6 was apparently upregulated, which contributes to active mTOR signaling in the sorafenib-resistant HCC organoids. Sorafenib resistance has been correlated with the upregulation of several signaling pathways including MTOR pathway as assayed by ribosomal protein $\mathrm{S} 6$ kinase phosphorylation in the tumor biopsies ${ }^{38}$. Interestingly, the expression of $\beta$-actin was downregulated in acquired sorafenib-resistant HCC-25. It has been reported that translation of human $\beta$ actin mRNA can be inhibited in the presence of mTOR kinase inhibitor ${ }^{39}$. Newly synthesized $\beta$-actin protein is indispensable for efficient cell migration ${ }^{14}$. The absence of $\beta$-actin protein might facilitate the retrodifferentiation of HCC organoids under the therapeutic pressure. Thus, phosphorylated ribosomal S6 kinase might be predictive for unresponsiveness of HCC to sorafenib and responsiveness of HCC to RTP.

Lastly, we confirmed in this study that the genes upregulated in the sorafenib-resistant HCC organoids were more associated with unfavorable postoperative prognosis in the TCGA databases than were the downregulated genes. These results are consistent with the data showing that cancer stemness- and EMT-related genes contribute to sorafenib resistance in HCC organoids, while stemness- and EMT-related genes are prognostic in $\mathrm{HCC}^{16,34-36}$. Our GSEA data also indicate that liver development- and liver specific gene-related gene sets were often downregulated, whereas undifferentiated cancer- and proliferation-related gene sets were upregulated in sorafenib-resistant HCC organoids (Figure 4B). These data indicate that a percentage of cancer cells retro-differentiated into stem-like cell types and obtain 
EMT feature are actively selected in sorafenib-presenting microenvironment and become more aggressive, sorafenib-resistant PLC. This process reflects the evolutionary and developmental nature of HCC during generating drug resistance against sorafenib pressure.

Conclusively, HCC organoids need a shorter time to construct and enrich more aggressive cancer cell types than does PDX. The majority of PLC organoids are resistant to lenvatinib in vitro, but they are sensitive to lenvatinib in the ODX models. Upregulations of stemness and EMT/partial EMT contribute to the acquired sorafenib resistance in HCC organoids, and targeting mTOR signaling pathway using mTOR inhibitor compound RTP is effective in treating acquired sorafenib-resistant HCC. Furthermore, phosphorylated ribosomal protein $\mathrm{S} 6$ should be a candidate predictive marker for the treatment of acquired sorafenib-resistant HCC with mTOR inhibitor(s). These novel findings might be applied to the future practice of cancer medicine.

\section{Materials And Methods}

\section{Patients and surgically removed cancer specimens}

Patients who received surgical resection of their tumors by Prof. Zhao's group at the Eastern Hepatobiliary Surgery Hospital (Shanghai, China) were included. Demographic information; pathological examinations including tumor nodule number, capsule integrity of tumor, and cancer embolus including $\mathrm{MVl}$; and the results of preoperative laboratory examinations including serum AFP, carbohydrate antigen 19-9 (CA19-9), HBV parameters, routine blood test, and liver function tests were extracted from electronic medical records. The clinical information of the included patients is listed in Table S6. This study was approved by the Ethics Committee of Eastern Hepatobiliary Surgery Hospital. Each patient provided an informed consent before the enrollment. Each surgically removed tumor specimen was divided into three parts. One part was processed to generate organoids and patient-derived xenograft (PDX). Another part was snap-frozen in liquid nitrogen for RNA extraction. The remaining tissue was fixed in $10 \%$ formalin and paraffin-embedded for histological and immunohistochemical analyses. For the culture of organoids, the samples were delivered to our laboratory at $4^{\circ} \mathrm{C}$ and processed immediately after excision. The pathological examination was performed thereafter.

\section{Culture of PLC organoids}

Tumor-derived organoids were cultured as follows. Briefly, tumor specimens were minced and incubated with the digestion solution at $37^{\circ} \mathrm{C}$. The suspension was filtered and centrifugally separated. The pellet was washed and then mixed with Matrigel (CORNING, Corning, NY). Cell clusters were then seeded into warmed-up 24-well plates. After approximately 15-20 min of solidification, organoid culture medium was added to the cells. Organoid medium contained advanced DMEM/F12 (Gibco, Billings, MT) supplemented with 1\% penicillin/streptomycin (GIBCO), 1\% glutamax (GIBCO), 10 mM HEPES (GIBCO), 1:50 B27 supplement (GIBCO), 1:100 N2 supplement (GIBCO), 1.25mM N-acetyl-L-cysteine (Sigma, St. Louis, MO), $10 \mathrm{mM}$ nicotinamide (Sigma), $50 \mathrm{ng} / \mathrm{mL}$ recombinant human epidermal growth factor (Peprotech, Cranbury, NJ), $100 \mathrm{ng} / \mathrm{mL}$ recombinant human fibroblast growth factor 10 (Peprotech), $25 \mathrm{ng} / \mathrm{mL}$ 
recombinant human hepatic growth factor (HGF) (Peprotech), $10 \mu \mathrm{M}$ forskolin (Selleckchemicals, Houston, TX), and $5 \mu \mathrm{M}$ A8301 (Selleckchemicals). Culture medium was changed twice a week. Organoids were split upon the attainment of dense culture and then frozen in liquid nitrogen.

\section{Xenografts of organoids and PDX models in nude mice}

All animal experiments including ODX were conducted were approved by the Institutional Animal Care and Use Committee of Second Military Medical University. For developing ODX, $1 \times 10^{6}$ organoids suspensions were prepared in $100 \mu \mathrm{L} 50: 50(\mathrm{v} / \mathrm{v})$ matrigel / culture medium and then injected subcutaneously into 6-8-week-old nude mice (Shanghai Jihui Laboratory, Shanghai, China) to build organoid-derived xenograft (ODX) models. PDX models were built as follows. The surgically removed tumor samples were collected in serum-free DMEM media within $30 \mathrm{~min}$ after resection and then cut into fragments $\left(20-30 \mathrm{~mm}^{3}\right)$ and implanted subcutaneously into the flanks of three-week-old male nude mice. The tumors were measured every two days using a caliper. When the tumor diameter reached $2 \mathrm{~cm}$, the mice were sacrificed. Tumors were weighted and excised into small pieces and then implanted into another group of nude mice. The remaining tumors were processed for histology and immunohistochemistry.

To assess the anti-tumor efficiency of sorafenib (LC Laboratories, Woburn, MA), regarofenib (LC Laboratories), lenvatinib (LC Laboratories), and RTP in vivo, mice were randomized by splitting sizematched tumors into five groups. The sorafenib/regarofenib/lenvatinib group received $30 \mathrm{mg} / \mathrm{kg}$ by gavage per day. In the RTP group, RAD001 $(2.5 \mathrm{mg} / \mathrm{kg})$ and TAK228 $(0.3 \mathrm{mg} / \mathrm{kg})$ were orally administrated by gavage and phenformin $(100 \mathrm{mg} / \mathrm{kg})$ was administrated by intraperitoneal injection every day as previously described ${ }^{40}$. The vehicle group received equal volume of $\mathrm{ddH}_{2} \mathrm{O}$. The tumor size was measured and recorded every three days with a caliper. The tumor volume was calculated with the formula Vol $=\left(L^{\prime} W^{2}\right) / 2$, where $L$ and $W$ are the long and short diameters of the tumor, respectively. The tumor weights were measured when mice were sacrificed.

\section{Histological analysis and immunohistochemistry}

Tumor tissues, organoids, ODX, and PDX were fixed in 4\% paraformaldehyde and embedded into paraffin blocks. Sections were subjected to H\&E and immunohistochemical examinations. An immunoreactive score with each antibody was evaluated as previously described ${ }^{41}$. The primary antibodies are listed in Table S7A.

\section{Inherent drug response of tumor organoids to targeted drugs}

The sensitivity of tumor organoids to each targeted drug was evaluated in vitro. Tumor organoids were digested and seeded at the density of $2-5 \times 10^{3}$ cells in $8 \mu \mathrm{L}$ Matrigel droplets. On Day 6 , each drug compound with a series of experimental concentrations was added to the cultures. The concentration ranges of the drugs were based on previously reported data ${ }^{7,40,42}$. Six days after treatment, cell viability was measured using Thiazolyl Blue Tetrazolium Bromide reagent (MTT, Sigma) in Synergy H1 Multi- 
Mode Reader (BioTek Instruments, Winooski, VT). Dimethyl sulfoxide (DMSO) (Wak Chemie Medical Gmbh, Steinbach/Ts, Germany)-treated tumor organoids were defined as $100 \%$ viability, and the results were normalized to the DMSO control. All experiments were performed in triplicate.

\section{Establishment of acquired sorafenib-resistant HCC organoids}

Tumor organoids from four HCC patients were randomly selected and cultured with sorafenib with an increasing concentration ranging $1.2-10 \mu \mathrm{M}$ (20\% per passaging) for about 3-5 months. The $\mathrm{IC}_{50}$ of sorafenib-resistant organoid was determined by MTT assay ${ }^{8}$. Sorafenib-resistant tumor organoids were then maintained in the medium with $4 \mu \mathrm{M}$ of sorafenib.

\section{RNA sequencing}

Total RNA was extracted from parental and their acquired sorafenib-resistant organoids using TRIzol (Invitrogen, Carlsbad, CA). RNA concentration and integrity were evaluated with the NanoDrop 2000 Spectrophotometer (Thermo Fisher Scientific, Waltham, MA) and Agilent 2100 Bioanalyzer (Agilent Technologies, Santa Clara, CA), respectively. Purified RNA was preserved at $-80{ }^{\circ} \mathrm{C}$. The libraries were constructed using TruSeq Stranded Total RNA with Ribo-Zero Gold (Illumina, San Diego, CA) and then sequenced on the Illumina NovaSeq 6000 sequencing platform (Illumina, San Diego, CA). Reads with 150 bp paired-ends were generated. The adapters were removed by Trimmomatic software ${ }^{43}$. The read pairs were mapped to reference genome of NCBI (GRCh38) by HISAT2 ${ }^{44}$. The transcriptomes were assembled by StringTie and compared with reference gene annotation by Cuffcompare software ${ }^{45,46}$. Finally, the read pairs were mapped to the pool of assembled transcripts by bowtie2 and the abundances of transcripts were estimated via performing eXpress ${ }^{47,48}$.

Differential gene expression was analyzed using the edgeR package $\mathrm{e}^{49}$. The genes with fold change $(F C) \geq 2$ and false discovery rate (FDR) $<0.05$ (obtained by Benjamini-Hochberg method) were considered as differentially expressed genes. The fold changes were collected to perform the "Prerank" method in gene set enrichment analysis (GSEA) ${ }^{50}$. Gene sets with false discovery rate (FDR) of $<0.01$ were considered significantly enriched. The original data of RNA-Seq in this study were deposited in the Gene Expression Omnibus (GEO) database with the accession GSE182593. The TCGA HCC (LIHC) dataset and the corresponding patients' clinical information were retrieved from the Xena website (https://xenabrowser.net).

\section{Western blotting}

Tumor organoids were released from the Matrigel by incubating with cell recovery solution (CORNING, Corning, NY) and then lysed with ice-cold Pierce RIPA Buffer with Halt Protease Inhibitor Cocktail (Thermo Fisher, Waltham, MA). The remaining steps were performed as previously described ${ }^{10}$. All antibodies are listed in Table S7B.

\section{Statistical analysis}


All statistical tests were performed using R platform (v 4.0.2) and GraphPad Prism v7 (GraphPad Software, San Diego, CA). The differences between two or multiple groups were determined by t-test or ANOVA. All tests were two-sided, and the threshold of a was 0.05 except when explicitly stated. The false discovery rate was calculated by Benjamini-Hochberg method. Curve fitting was performed using GraphPad Prism software and nonlinear regression equation.

\section{Declarations}

\section{Funding:}

This work was supported by grant 2015CB554006 from the National Key Basic Research Program of China (GC); grants 91529305 (GC), 81520108021 (GC), 81673250 (GC), 81521091 (GC), 82003538 (WL), and 81502882 (XC) from the National Natural Science Foundation of China; grant 20 YF1458800 (WL) from the Shanghai Yangfan Program; and grants GWV-10.1-XK17 from the "3-year public health promotion" program of Shanghai Municipal Health Commission (GC).

\section{Author contributions}

Cao G conceived and supervised the study. Xian L, Zhao P, and Wei Z were responsible for the culture and maintenance of tumor organoids. Chen $\mathrm{X}$ did the bioinformatics analysis. Ji $\mathrm{H}$ and Zhao $\mathrm{J}$ did surgical treatments and provided suitable clinical specimens. Liu D, Li Z, Liu W, Zhou X, Fan J, Zhu X, Yin J, and Tan $\mathrm{X}$ took parts in cell experiment and animal care. Qian $\mathrm{Y}$ and Dong $\mathrm{H}$ took part in histology and immunohistochemistry assays. Chen $\mathrm{X}$, $\operatorname{Han} \mathrm{X}$, and $\mathrm{Yu} \mathrm{H}$ conducted the statistical analyses. Cao $\mathrm{G}$ interpreted the data and drafted the manuscript. All authors read and approved the final manuscript.

Competing interests: Authors declare that they have no competing interests.

Data and materials availability: The original data of RNA-Seq in this study were deposited in the Gene Expression Omnibus (GEO) database with the accession GSE182593.

\section{References}

1. Sung $\mathrm{H}$ et al. Global Cancer Statistics 2020: GLOBOCAN Estimates of Incidence and Mortality Worldwide for 36 Cancers in 185 Countries. CA Cancer J Clin 71,209-249(2021).

2. Jiang D et al. Trends in cancer mortality in China from 2004 to 2018: A nationwide longitudinal study. Cancer Commun (Lond) 41,1024-1036(2021).

3. Yang F et al. Contribution of Hepatitis B Virus Infection to the Aggressiveness of Primary Liver Cancer: A Clinical Epidemiological Study in Eastern China. Front Oncol 9:370(2019).

4. Marasco $\mathrm{G}$ et al. Predictive factors for hepatocellular carcinoma recurrence after curative treatments. Hepatoma Research 6:333(2020). 
5. Rammohan A \& Rela M. Risk factors and management of post-liver transplant recurrence of hepatocellular carcinoma. Hepatoma Research 7:49(2020).

6. Lo YH, Karlsson K \& Kuo CJ. Applications of Organoids for Cancer Biology and Precision Medicine. Nat Cancer 1:761-773(2020).

7. Broutier $L$ et al. Human primary liver cancer-derived organoid cultures for disease modeling and drug screening. Nat Med 23:1424-35(2017).

8. Nuciforo S et al. Organoid Models of Human Liver Cancers Derived from Tumor Needle Biopsies. Cell Rep 24(2018)

9. Li L et al. Human primary liver cancer organoids reveal intratumor and interpatient drug response heterogeneity. JCI Insight 4(2019).

10. Wang $S$ et al. Hedgehog signaling promotes sorafenib resistance in hepatocellular carcinoma patient-derived organoids. J Exp Clin Cancer Res 39:22(2020).

11. Zhao $Y$ et al. Single-Cell Transcriptome Analysis Uncovers Intratumoral Heterogeneity and Underlying Mechanisms for Drug Resistance in Hepatobiliary Tumor Organoids. Adv Sci (Weinh) 8:e2003897(2021).

12. Leung CON et al. Overriding Adaptive Resistance to Sorafenib Through Combination Therapy With Src Homology 2 Domain-Containing Phosphatase 2 Blockade in Hepatocellular Carcinoma. Hepatology 72:155-68(2020).

13. Ippen FM et al. The Dual PI3K/mTOR Pathway Inhibitor GDC-0084 Achieves Antitumor Activity in PIK3CA-Mutant Breast Cancer Brain Metastases. Clin Cancer Res 25:3374-83(2019).

14. Kim NY, et al. Optogenetic control of mRNA localization and translation in live cells. Nat Cell Biol 22:341-352(2020).

15. Yin $\mathrm{J}$ et al. Effect of antiviral treatment with nucleotide/nucleoside analogs on postoperative prognosis of hepatitis B virus-related hepatocellular carcinoma: a two-stage longitudinal clinical study. J Clin Oncol 31:3647-3655(2013).

16. Kawai T et al. Keratin 19, a Cancer Stem Cell Marker in Human Hepatocellular Carcinoma. Clin Cancer Res 21:3081-3091(2015).

17. Kuo YH et al. Nivolumab Versus Regorafenib in Patients With Hepatocellular Carcinoma After Sorafenib Failure. Front Oncol 11:683341(2021).

18. Zhu M et al. Uncovering Biological Factors That Regulate Hepatocellular Carcinoma Growth Using Patient-Derived Xenograft Assays. Hepatology 72:1085-101(2020).

19. Zhang $Q$ et al. Lenvatinib promotes antitumor immunity by enhancing the tumor infiltration and activation of NK cells. Am J Cancer Res 9:1382-95(2019).

20. Carroll JL, et al. The role of natural killer cells in adenovirus-mediated p53 gene therapy. Mol Cancer Ther 1:49-60 (2001).

21. Llovet JM et al. Sorafenib in advanced hepatocellular carcinoma. N Engl J Med 359:378-90(2008). 
22. Zhu Q et al. Silencing KIF14 reverses acquired resistance to sorafenib in hepatocellular carcinoma. Aging (Albany NY) 12:22975-3003(2020).

23. Tovar $V$ et al. Tumour initiating cells and IGF/FGF signalling contribute to sorafenib resistance in hepatocellular carcinoma. Gut 66:530-40(2017).

24. Liu M et al. MCM6 promotes metastasis of hepatocellular carcinoma via MEK/ERK pathway and serves as a novel serum biomarker for early recurrence. J Exp Clin Cancer Res 37:10 (2018).

25. Wang $\mathrm{J}$ et al. Knockdown of RRS1 by lentiviral-mediated RNAi promotes apoptosis and suppresses proliferation of human hepatocellular carcinoma cells. Oncol Rep 38:2166-72(2017).

26. Zhou Z et al. TP53INP2 Modulates Epithelial-to-Mesenchymal Transition via the GSK-3 $\beta / \beta-$ Catenin/Snail1 Pathway in Bladder Cancer Cells. Onco Targets Ther 13:9587-97(2020).

27. Surcel A et al. Targeting Mechanoresponsive Proteins in Pancreatic Cancer: 4-Hydroxyacetophenone Blocks Dissemination and Invasion by Activating MYH14. Cancer Res 79:4665-78(2019).

28. Zhang $B$ et al. The splicing regulatory factor hnRNPU is a novel transcriptional target of c-Myc in hepatocellular carcinoma. FEBS Lett 595:68-84(2021).

29. Talukdar S, Emdad L, Das SK \& Fisher PB. EGFR: An essential receptor tyrosine kinase-regulator of cancer stem cells. Adv Cancer Res 147:161-88(2020).

30. Chen $\mathrm{J}$ et al. DEPTOR induces a partial epithelial-to-mesenchymal transition and metastasis via autocrine TGF $\beta 1$ signaling and is associated with poor prognosis in hepatocellular carcinoma. J Exp Clin Cancer Res 38:273(2019).

31. Xing J et al. Haprolid Inhibits Tumor Growth of Hepatocellular Carcinoma through Rb/E2F and Akt/mTOR Inhibition. Cancers (Basel) 12:615 (2020).

32. Zhu $L$ et al. LncRNA CRNDE promotes the epithelial-mesenchymal transition of hepatocellular carcinoma cells via enhancing the Wnt/ $\beta$-catenin signaling pathway. J Cell Biochem 120:115664(2018).

33. Stebbing J, Filipović A \& Giamas G. Claudin-1 as a promoter of EMT in hepatocellular carcinoma. Oncogene 32:4871-2(2013).

34. Hu M, Li M, Huang H \& Lu C. Isolated cancer stem cells from human liver cancer: morphological and functional characteristics in primary culture. Clin Transl Oncol 24:(2021).

35. Sang $X$ et al. Human Hepatic Cancer Stem Cells (HCSCs) Markers Correlated With Immune Infiltrates Reveal Prognostic Significance of Hepatocellular Carcinoma. Front Genet 11:112(2020).

36. Pastushenko I \& Blanpain C. EMT Transition States during Tumor Progression and Metastasis. Trends Cell Biol 29:212-26(2019).

37. Pastushenko I et al. Identification of the tumour transition states occurring during EMT. Nature 556:463-8(2018).

38. Masuda $\mathrm{M}$ et al. Alternative mammalian target of rapamycin (mTOR) signal activation in sorafenibresistant hepatocellular carcinoma cells revealed by array-based pathway profiling. Mol Cell Proteomics 13:1429-38(2014). 
39. Eliseeva I, Vasilieva M \& Ovchinnikov LP. Translation of Human $\beta$-Actin mRNA is Regulated by mTOR Pathway. Genes (Basel) 10:96(2019).

40. Veiga SR et al. Phenformin-Induced Mitochondrial Dysfunction Sensitizes Hepatocellular Carcinoma for Dual Inhibition of mTOR. Clin Cancer Res. 24:3767-80(2018).

41. Chang W et al. Gene expression profiling-derived immunohistochemistry signature with high prognostic value in colorectal carcinoma. Gut 63:1457-67(2014).

42. Rodríguez-Hernández MA et al. Differential effectiveness of tyrosine kinase inhibitors in 2D/3D culture according to cell differentiation, p53 status and mitochondrial respiration in liver cancer cells. Cell Death Dis 11:339(2020).

43. Bolger AM, Lohse M \& Usadel B. Trimmomatic: a flexible trimmer for Illumina sequence data. Bioinformatics 30:2114-20(2014).

44. Kim D, Langmead B \& Salzberg SL. HISAT: a fast spliced aligner with low memory requirements. Nat Methods 12:357-60(2015).

45. Pertea $\mathrm{M}$ et al. StringTie enables improved reconstruction of a transcriptome from RNA-seq reads. Nat Biotechnol 33:290-5(2015).

46. Trapnell $C$ et al. Differential gene and transcript expression analysis of RNA-seq experiments with TopHat and Cufflinks. Nat Protoc 7:562-78(2012).

47. Langmead B \& Salzberg SL. Fast gapped-read alignment with Bowtie 2. Nat Methods 9:3579(2012).

48. Roberts A \& Pachter L. Streaming fragment assignment for real-time analysis of sequencing experiments. Nat Methods 10:71-3(2013).

49. McCarthy DJ, Chen Y \& Smyth GK. Differential expression analysis of multifactor RNA-Seq experiments with respect to biological variation. Nucleic Acids Res 40:4288-97(2012).

50. Subramanian A et al. Gene set enrichment analysis: a knowledge-based approach for interpreting genome-wide expression profiles. Proc Natl Acad Sci U S A 102:15545-50(2005).

\section{Table}

Table 1. Baseline characteristics of 131 HCC patients from whom tumor organoids were developed. 


\begin{tabular}{|c|c|c|c|}
\hline \multirow[t]{2}{*}{ Variable } & \multicolumn{2}{|c|}{ No. of Patients } & \multirow[t]{2}{*}{$\mathrm{P}$-value } \\
\hline & $\begin{array}{l}\text { Successfully } \\
\text { developing organoid }\end{array}$ & $\begin{array}{l}\text { Unsuccessfully } \\
\text { developing organoid }\end{array}$ & \\
\hline All cases & 38 & 93 & \\
\hline $\begin{array}{l}\text { Age (year) > } 50 \text { vs. } \\
\leq 50\end{array}$ & $17: 21$ & $63: 30$ & $0.014^{*}$ \\
\hline $\begin{array}{l}\text { Gender male vs. } \\
\text { female }\end{array}$ & $31: 7$ & $84: 9$ & 0.274 \\
\hline HBsAg + vs. - & $34: 4$ & $79: 14$ & 0.495 \\
\hline HBeAg + vs. - & $10: 28$ & $22: 71$ & 0.748 \\
\hline $\begin{array}{l}\text { Liver cirrhosis + vs. } \\
\text { - }\end{array}$ & $20: 18$ & 41:52 & 0.374 \\
\hline $\begin{array}{l}\text { Liver function Child } \\
\text { A vs. B }\end{array}$ & $36: 2$ & $89: 4$ & 1.000 \\
\hline $\begin{array}{l}\operatorname{AFP}(\mu \mathrm{g} / \mathrm{L}) \\
>20: \leq 20\end{array}$ & $30: 8$ & $51: 42$ & $0.010^{*}$ \\
\hline $\begin{array}{l}\text { Tumor size }(\mathrm{cm}) \\
>5: \leq 5\end{array}$ & $30: 8$ & $47: 46$ & $0.003^{*}$ \\
\hline $\begin{array}{l}\text { No. tumor nodule: } \\
\text { Solitary vs. multiple }\end{array}$ & $32: 6$ & 78:15 & 0.962 \\
\hline $\begin{array}{l}\text { Differentiation I+II } \\
\text { vs. III+IV }\end{array}$ & $0: 38$ & $5: 88$ & 0.340 \\
\hline $\begin{array}{l}\text { Micro vascular } \\
\text { invasion } \\
\text { + vs. - }\end{array}$ & $31: 7$ & $55: 38$ & $0.014^{*}$ \\
\hline $\begin{array}{l}\text { Macro vascular } \\
\text { invasion } \\
\text { + vs. - }\end{array}$ & $11: 27$ & 4:89 & $0.000^{*}$ \\
\hline $\begin{array}{l}\text { TNM stage I vs. } \\
\text { II+III+IV }\end{array}$ & $5: 33$ & $35: 58$ & $0.006^{*}$ \\
\hline $\begin{array}{l}\text { BCLC stage 0-A vs. } \\
\text { B-C }\end{array}$ & $2: 36$ & $23: 70$ & $0.010^{*}$ \\
\hline
\end{tabular}

\section{Figures}




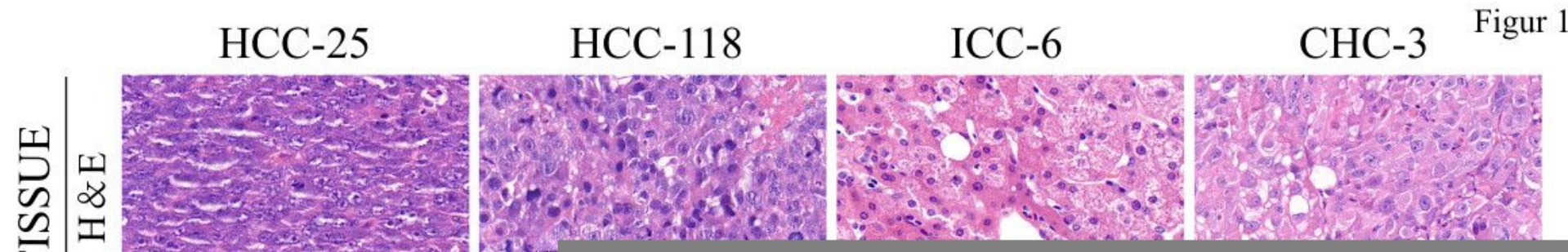

\section{Figure 1}

Histology of primary tumor tissues, tumor organoids, ODX, and PDX from HCC-25, HCC-118, ICC-6, and $\mathrm{CHC}-3$ patients. H\&E staining of the tumor tissues, bright-field and H\&E staining of tumor organoids originated from the same corresponding tissues, H\&E staining of ODX, and H\&E staining of PDX are shown from the top row to the bottom row. Scale bars, $50 \mu \mathrm{m}$. 
A

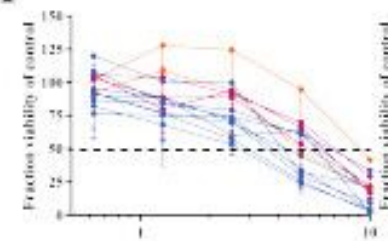

B

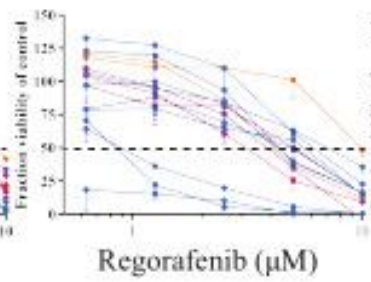

Regorafenib $(\mu \mathrm{M})$

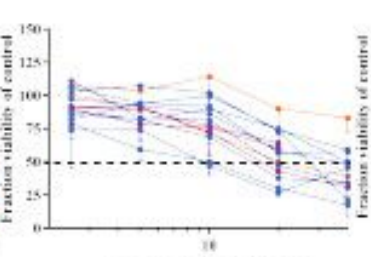

Lenvatinib ( $\mu \mathrm{M})$

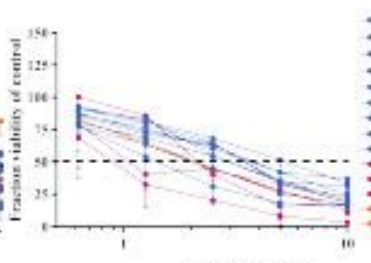

RTP (nM)

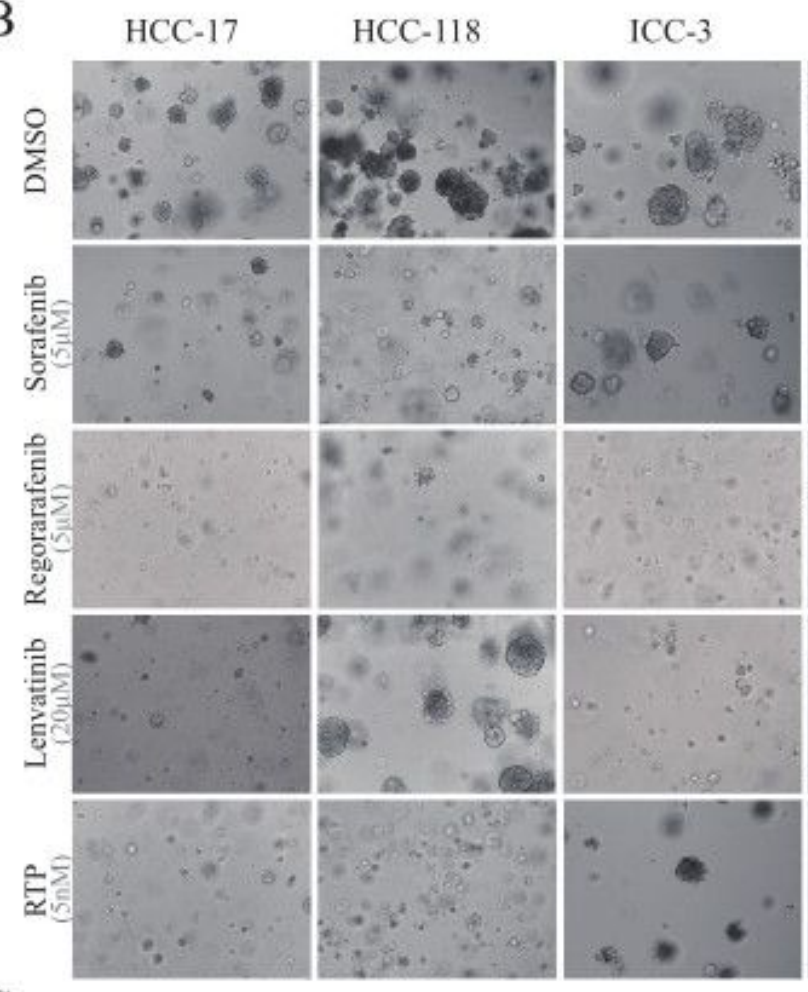

ICC-13

CHC-3

$\mathrm{CHC}-4$
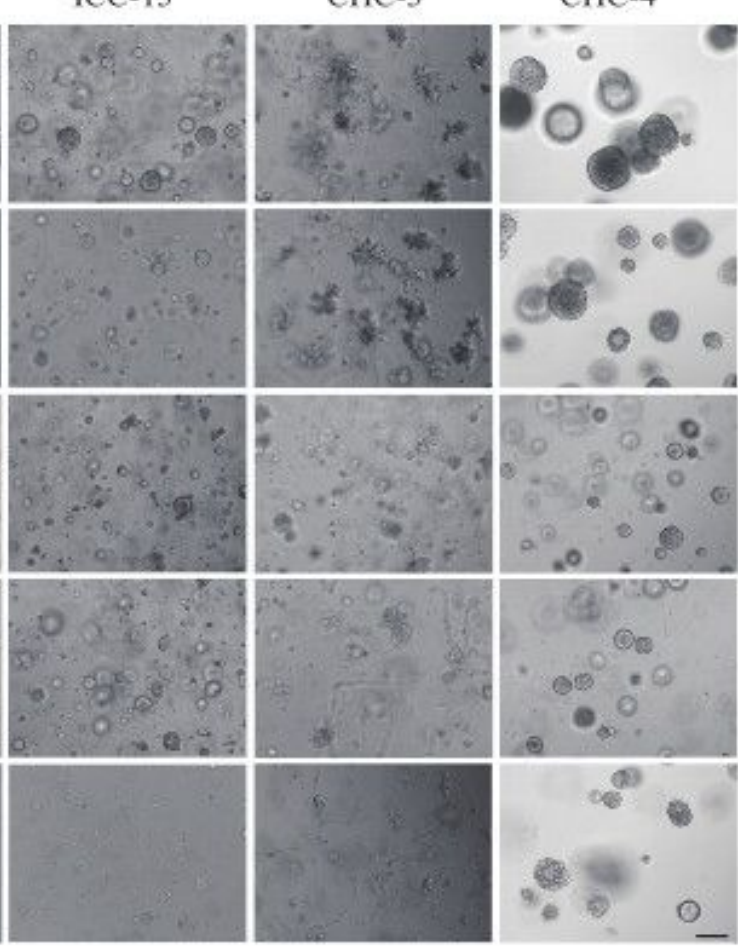

$\mathrm{C}$

HCC-17

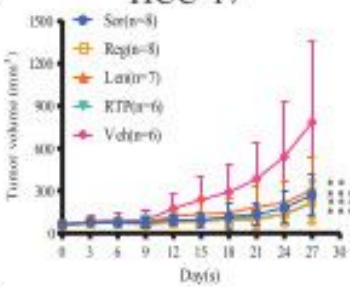

HCC-118

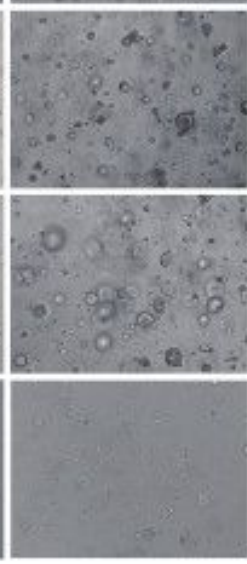

ICC- 3

CHC-3
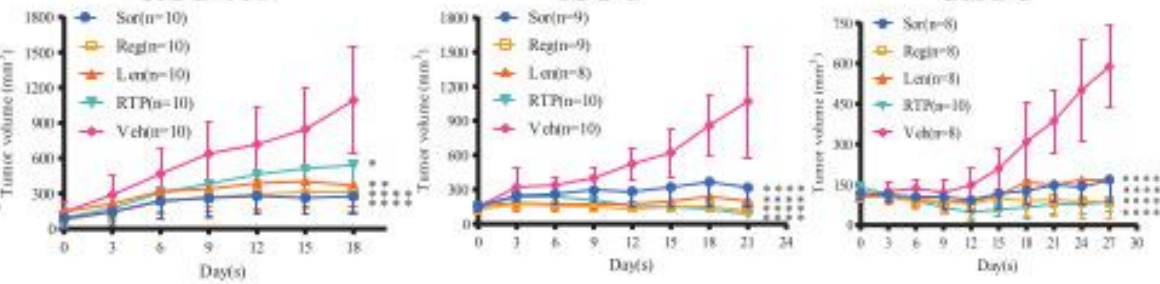

$\mathrm{D}$

HCC-17

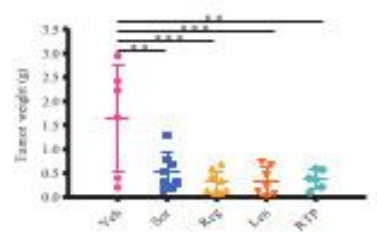

HCC-118
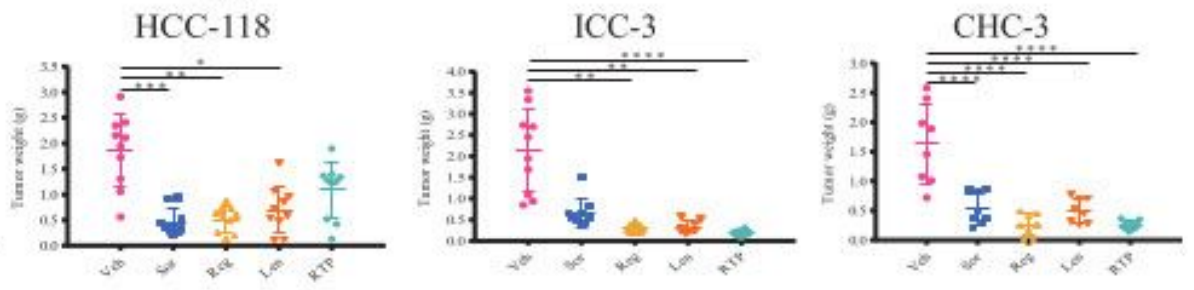

\section{Figure 2}

The sensitivities of PLC organoids to targeted drugs in vitro and in vivo. (A) Response of the HCC, ICC, and $\mathrm{CHC}$ organoids to sorafenib, regorafenib, lenvatinib, and RTP treatment for six days, respectively. Data displayed are the average values of three technical and three biological repeats. (B) Bright-field images of HCC-17, HCC-118, ICC-3, ICC-13, CHC-3, and CHC-4 organoids after being in vitro treated with sorafenib $(5 \mu \mathrm{M})$, regorarafenib $(5 \mu \mathrm{M})$, lenvatinib $(20 \mu \mathrm{M})$, and RTP $(5 \mathrm{nM})$ on Day 6 , respectively. Scale 
bars, $50 \mu \mathrm{m}$. (C) Tumor volume of HCC-17, HCC-118, ICC-3, and CHC-3 ODX during the treatment with sorafenib, regorarafenib, lenvatinib, and RTP, respectively. (D) Tumor weight of HCC-17, HCC-118, ICC-3, and $\mathrm{CHC}-3 \mathrm{ODX}$ at the end of the treatment with sorafenib, regorarafenib, lenvatinib, and RTP, respectively.

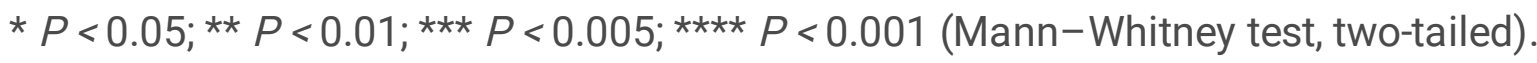

Figure 3

A

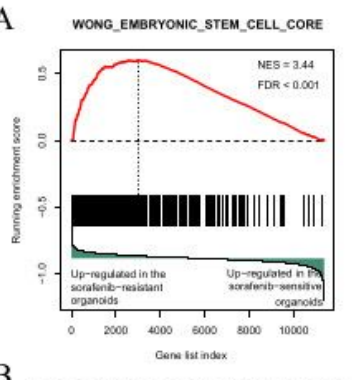

B

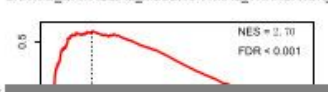

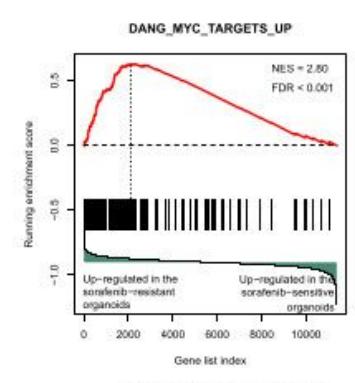

KARLSSON_TGFB1_TARGETS_UP

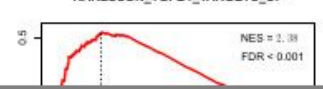

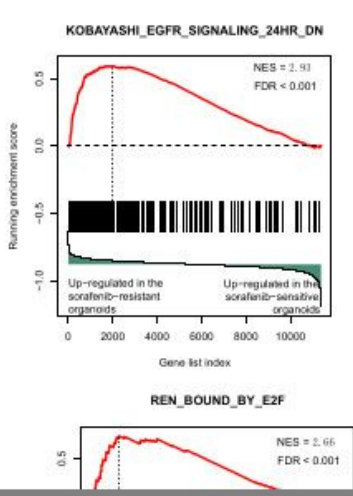

C

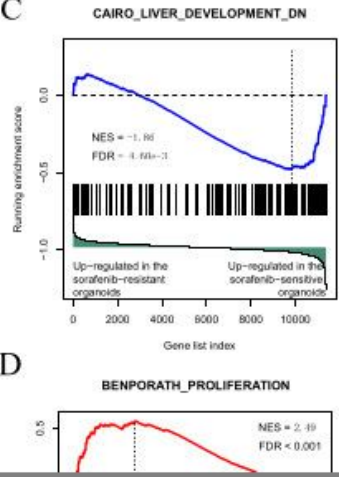

HSIAO_LIVER_SPECIFIC_GENES
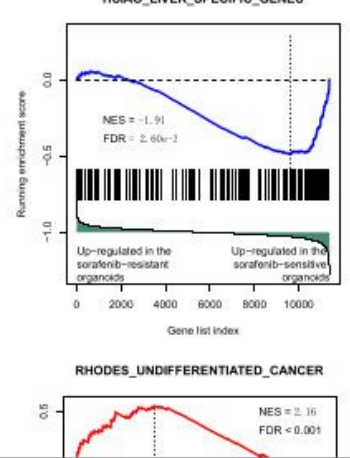

\section{Figure 3}

Representative gene sets enriched in acquired sorafenib-resistant HCC organoids: (A) gene sets representing the signature of cancer stemness; (B) gene sets representing the signature of EMT; (C) gene sets representing the signature of liver development; and (D) gene sets representing the signature of tumor undifferentiation. EMT, epithelial-mesenchymal transition; NES, normalized enrichment score; FDR, false discovery rate. 
Figure 4

A
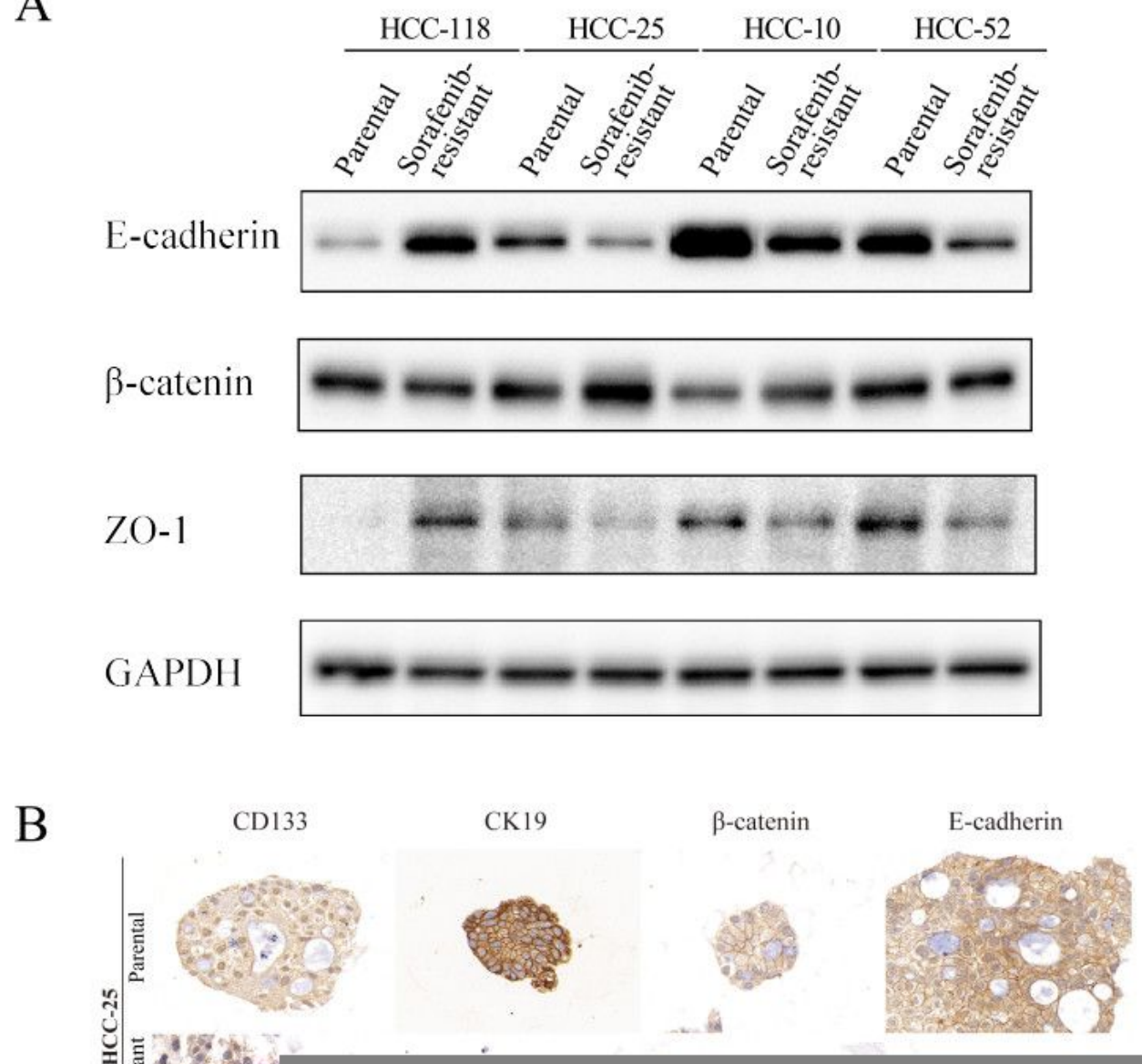

\section{Figure 4}

The expression of cancer stemness- and EMT-related genes between parental and sorafenib-resistant HCC organoids. (A) Western blotting showed the expression patterns of E-cadherin, $\beta$-catenin, and ZO- 1 in the four sorafenib-resistant HCC organoids and parental ones. The expression of GAPDH was applied as the loading control. (B) Immunohistochemistry showed the expression patterns of CD133, CK-19, $\beta$ - 
catenin, and E-cadherin in parental and acquired sorafenib-resistant HCC-25 and HCC-118 organoids. Scale bars, $50 \mu \mathrm{m}$.

Figure 5

A

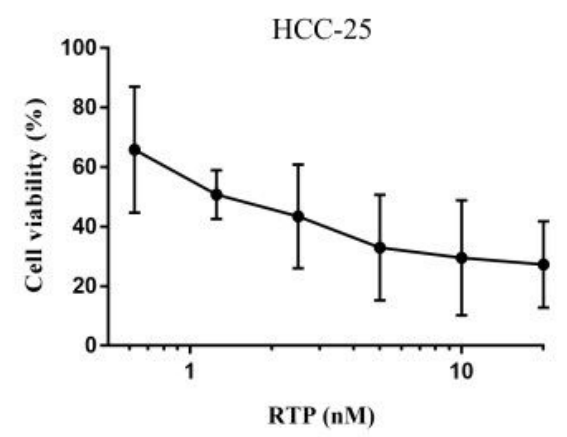

D

HCC-25

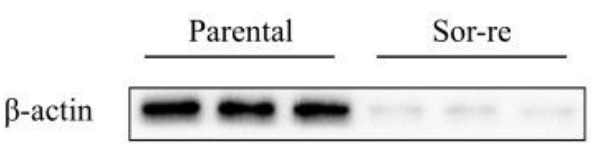

GAPDH
B

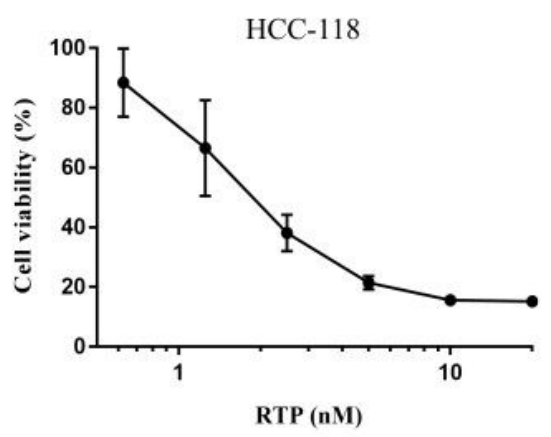

E

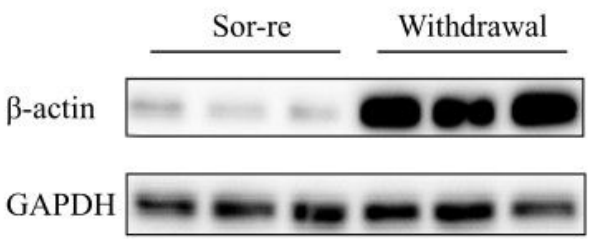

C

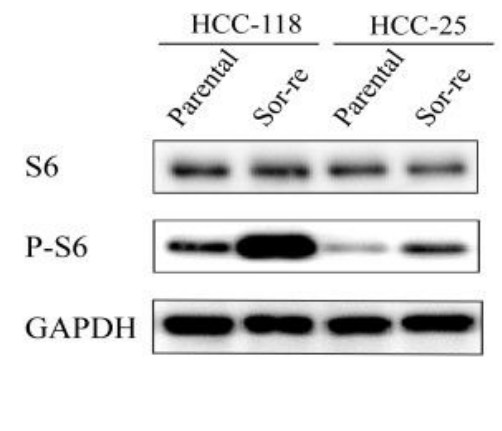

F

HCC-118

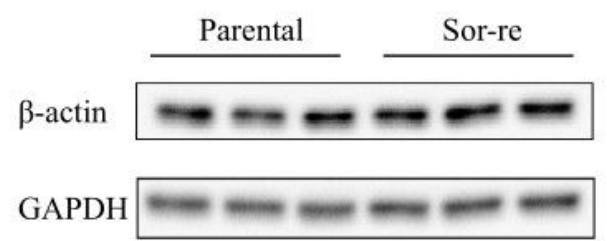

\section{Figure 5}

The effects of mTOR signaling pathway inhibitor on the viability of acquired sorafenib-resistant HCC organoids and its possible mechanisms. (A) The response of acquired sorafenib-resistant HCC-25 organoids to the treatment with RTP for six days. (B) The response of acquired sorafenib-resistant HCC118 organoids to the treatment with RTP for six days. (C) The expression of S6, one of the downstream effectors of the mTOR pathway, and phosphorylation of S6 (P-S6) in parental and acquired sorafenibresistant organoids HCC-118 and HCC-25 in Western blot. (D) Western blotting revealed the downregulation of $\beta$-actin expression in acquired sorafenib-resistant organoid HCC-25. (E) Recovery of $\beta$ actin expression in HCC-25 organoids after the withdrawal of sorafenib. (F) The expression of $\beta$-actin was not affected in sorafenib-resistant HCC-118 organoids. The expression level of GAPDH was used as the loading control.

\section{Supplementary Files}

This is a list of supplementary files associated with this preprint. Click to download.

- SupplementaryMaterial.docx 
- FigureS1.pdf

- Figures2.pdf

- FigureS3.pdf

- FigureS4.pdf

- Figures5.pdf

- Tables1.docx

- Tables2.docx

- Tables3.docx

- Tables4.docx

- TableS5.docx

- TableS6.xlsx

- TableS7.docx 